Quim. Nova, Vol. 35, No. 2, 274-279, 2012

\title{
ASPECTOS ANALÍTICOS DA RESPOSTA DO DETECTOR DE IONIZAÇÃO EM CHAMA PARA ÉSTERES DE ÁCIDOS GRAXOS EM BIODIESEL E ALIMENTOS
}

\author{
Jesui Vergilio Visentainer \\ Departamento de Química, Universidade Estadual de Maringá, Av. Colombo, 5790, 87020-900 Maringá - PR, Brasil
}

Recebido em 11/1/11; aceito em 6/7/11; publicado na web em 19/8/11

\begin{abstract}
ANALYTICAL ASPECTS OF THE FLAME IONIZATION DETECTOR RESPONSE OF FATTY ACID ESTERS IN BIODIESELS AND FOODS. The analysis of fatty acid (FA) esters by gas chromatography and flame ionization detector (FID) normally uses the normalization method. However, if one FA is wrongly estimated, the results could be greatly affected. In this study, methodologies using internal standards and correction factors for the FID response are described. The results show that by using theoretical correction factors associated to the internal standardization, the quantitative analyses of the FAs are expressed in mass, increasing the accuracy and facilitating the interpretation and comparison of the results for foods and biodiesels.
\end{abstract}

Keywords: biodiesel; oil; flame ionization detector response.

\section{INTRODUÇÃO}

Diferentes áreas da ciência têm utilizado as avaliações qualitativas e quantitativas de ésteres de ácidos graxos (EAGs) nas determinações analíticas, em estudos de diferentes componentes lipídicos. Nos últimos anos, as áreas de alimentos, bioquímica e de biodiesel têm se destacado nos estudos dos ácidos graxos e de EAGs. A formação destes ésteres comumente ocorre da reação entre óleos e gorduras (lipídios) com monoalcoóis de cadeia curta, como o metanol ou o etanol, os quais fornecem o radical metil e etil, respectivamente. Quando a reação é com o metanol, formam-se os ésteres metílicos de ácidos graxos (EMAGs), um dos mais estudados, no entanto, quando a reação é com o etanol, formam-se os ésteres etílicos de ácidos graxos. ${ }^{1,2}$ A competição entre a utilização do metanol versus etanol, nas reações de obtenção dos EAGs, tem origens em vários fatores, dentre estes a disponibilidade, o rendimento de reação, o preço, a reatividade, a toxidez, a poluição etc.

Estudos com ácidos graxos na área de alimentos e bioquímica, comumente, utilizam EMAGs na avaliação de alimentos funcionais, tais como, a composição em ácidos graxos em carnes, ${ }^{3,4}$ ácidos graxos ômega-3,,$^{5,6}$ manipulação de ácidos graxos em animais ${ }^{7}$ e de ácidos graxos maléficos à saúde humana (ácidos graxos trans). ${ }^{8,9}$ Da mesma forma, têm sido realizados estudos com biodiesel no Brasil para avaliar as matérias-primas, ${ }^{1}$ catalisadores, ${ }^{10}$ padrão interno ${ }^{11}$ e os EAGs. ${ }^{7,9}$

Ressalta-se que o denominador comum, independentemente da origem da matéria-prima alimentícia ou matriz para biodiesel, da reação de transesterificação ou da reação de esterificação, é a concentração dos EAGs, ou seja, esses componentes podem ser utilizados como biodiesel ou analitos para avaliar a composição em ácidos graxos dos alimentos. As insaturações e o tamanho da cadeia hidrocarbônica refletem diretamente no número de carbonos ativos e, consequentemente, são fatores decisivos nas reações que ocorrem no detector de ionização em chama (DIC). ${ }^{12}$

O DIC apresenta uma quantidade mínima detectável de aproximadamente $10^{-12} \mathrm{~g}$ (para alcanos), uma resposta quase universal, faixa de linearidade ampla, é simples de operar e de resposta rápida. ${ }^{13}$ Neste artigo, são apresentadas as discussões para os EMAGs, embora o estudo possa ser estendido para todos os EAGs (ésteres etílicos, propílicos etc.).

\footnotetext{
e-mail: jvvisentainer.uem.br
}

O DIC é sensível ao fluxo de massa e a reação abaixo mostra a ionização química que ocorre no detector de ionização em chama: ${ }^{14}$

$$
\mathrm{CH}+\mathrm{O} \rightarrow \mathrm{CHO}^{+}+\mathrm{e}^{-}
$$

O íon $\mathrm{CHO}^{+}$é instável e reage rapidamente com água na chama para gerar o hidroxônio, conforme a reação:

$$
\mathrm{CHO}^{+}+\mathrm{H}_{2} \mathrm{O} \rightarrow \mathrm{H}_{3} \mathrm{O}^{+}+\mathrm{CO}
$$

Esta reação ocorre para 100.000 átomos de carbono introduzidos na chama. Portanto, a resposta do DIC é proporcional ao número de átomos de carbono que é queimado. ${ }^{12,14}$

A magnitude do sinal gerado pelo DIC é proporcional ao número de átomos de carbono e hidrogênio na molécula que está sendo analisada, ${ }^{13}$ ou seja, é proporcional aos átomos de carbonos ligados a átomos de hidrogênios (carbono ativo $\mathrm{C}^{*}$ ). No entanto, existem fatores intrínsecos de uma determinada molécula de EMAG que afetam a resposta do DIC, dentre estes, a presença de oxigênio, que diminui a resposta do DIC. ${ }^{13,14} \mathrm{O}$ átomo de carbono do grupo carboxilato (COO) não é ionizado, apreciavelmente, durante a combustão e não é considerado como carbono ativo. ${ }^{15-17}$ Além disso, a existência de diferentes efeitos na combustão, entre cadeia saturada e insaturada, ocorre devido à redução de átomos de hidrogênio nas duplas ligações. ${ }^{16}$

No intuito de obter maior exatidão na quantificação, deve-se considerar que os EMAGs respondem diferencialmente no DIC. Desta forma, é necessário utilizar fator de correção experimental ou empírico $\left(\mathrm{F}_{\mathrm{CE}}\right)$, ou fator de correção teórico $\left(\mathrm{F}_{\mathrm{CT}}\right)$, determinado teoricamente a partir do número de carbonos ativos $\left(\mathrm{C}^{*}\right)$. Ambos os fatores são obtidos baseando-se em comparações entre os EMAGs e um padrão interno. ${ }^{12}$

O método da padronização interna ( $\mathrm{Pi}$ ) é indicado, considerandose que é menos sensível a erros de injeções e variações instrumentais, além disso o padrão interno deve apresentar os requisitos de não estar presente na amostra, ser acessível e barato, apresentar alto grau de pureza, ser adicionado em concentrações próxima aos analitos, eluir separadamente dos componentes da amostra, apresentar boa estabilidade etc. ${ }^{12}$ É evidente que todos estes requisitos são difíceis de serem contemplados nas análises de uma mistura de EMAGs. No entanto, alguns ésteres têm sido recomendados como, por exemplo, 
o tricosanoato de metila (23:0Me) para alimentos ${ }^{18}$ e heptadecanoato de metila (17:0Me) e oleato de etila (18:1Et) para biodiesel. ${ }^{11}$ Estes padrões internos são preferencialmente utilizados por apresentarem completa resolução entre os picos, não estarem presentes na amostra e a maioria apresentar boa estabilidade por serem saturados; evidentemente, outros padrões podem ser utilizados na dependência da composição da amostra.

No Brasil, os trabalhos realizados nas análises de EMAGs sejam para alimentos ou para biodiesel, normalmente utilizam o método da normalização de área, onde os resultados são expressos em porcentagem de área relativa. ${ }^{12,19,20}$ As desvantagens da normalização são as propagações de erros devido à interdependência dos resultados. Na normalização de área, todos os componentes da amostra devem ser detectados e, na ocorrência de omissão ou estimativa de um componente, a área dos demais componentes é afetada. Além disso, os resultados apresentados por este método apresentam dificuldades de interpretação e muitas vezes são publicados de forma errônea. ${ }^{12,21}$

Por outro lado, na expressão dos resultados de EMAGs (biodiesel) ou de ácidos graxos (alimentos), onde os resultados das concentrações são expressos em massa de EMAG/massa de matéria-prima ou de amostra, tanto o uso de padrão interno como de fatores de correção do detector de ionização em chama proporcionarão resultados com maior exatidão e, portanto, resultados facilmente interpretados que poderão ser utilizados por profissionais das diferentes áreas sem maiores dificuldades. ${ }^{12,21}$ Ressalta-se que, na dificuldade de utilização de fatores de correção, deva-se usar padronização interna e, desta foram, expressar os resultado em concentração e não em porcentagem de área.

O propósito do presente estudo foi primeiramente instruir e demonstrar o uso de técnicas analíticas apropriadas e que apresentam maior exatidão na expressão dos resultados da concentração de ácidos graxos (em alimentos), ésteres de ácidos graxos (biodiesel) e aplicar as técnicas descritas em uma amostra de óleo de peixe. Desta forma, além de mostrar a aplicação da técnica, que pode ser estendida para qualquer amostra lipídica, os resultados expressos de concentração de EMAGS ou ácidos graxos apresentam maior exatidão e podem ser facilmente interpretados.

\section{PARTE EXPERIMENTAL}

\section{Extração de lipídios totais e umidade}

Um volume de aproximadamente $10,0 \mathrm{~mL}$ de óleo de peixe foi obtido da extração de filés de tilápias (5 lotes de 200 g de filé/lote, rendimento médio de $2 \mathrm{~g}$ de óleo/lote), de acordo com o método de Bligh e Dyer, ${ }^{22}$ usando clorofórmio:methanol:água nas razões de 1:2:0,8 (na primeira etapa) e de 2:2:1 (na etapa final). A umidade foi determinada conforme método da AOAC (Association of Official Analytical Chemists). ${ }^{23}$

\section{Condições cromatográficas}

Os EMAGs foram preparados conforme descrito no método de Joseph e Ackman ${ }^{18}$ e separados em cromatógrafo a gás Varian 3300 (Palo Alto, USA) acoplado com DIC e coluna de sílica fundida DB-WAX- J \& W Scientific, Folsom, CA, USA (30 m x 0,25 $\mu \mathrm{m}$ de espessura do filme e $0,25 \mathrm{~mm}$ de d.i.). ${ }^{24}$ Os parâmetros operacionais foram: temperaturas do detector e injetor de 280 e $250{ }^{\circ} \mathrm{C}$, respectivamente. A temperatura da coluna de $170{ }^{\circ} \mathrm{C}$ por $16 \mathrm{~min}$ foi programada a $2{ }^{\circ} \mathrm{C} \mathrm{min}{ }^{-1}$ até $210^{\circ} \mathrm{C}$ e mantida nesta temperatura por 25 min. $\mathrm{O}$ gás de arraste foi $\mathrm{H}_{2}$, com fluxo de $0,8 \mathrm{~mL} \mathrm{~min}^{-1}$ e velocidade linear de $38 \mathrm{~cm} \mathrm{~s}^{-1}$ com filtro de oxigênio acoplado na linha de alimentação. $\mathrm{O}$ gás auxiliar (make-up) foi $\mathrm{N}_{2}$ a $30 \mathrm{~mL} \mathrm{~min}^{-1}$, a chama do DIC produzida com $\mathrm{H}_{2}\left(30 \mathrm{~mL} \mathrm{~min}^{-1}\right)$ e ar sintético (300 $\mathrm{mL} \min ^{-1}$ ) e a divisão de amostra (split) de 1:50. Todas as etapas da reação de metilação foram realizadas sob atmosfera de $\mathrm{N}_{2}$ Os tempos de retenção dos analitos e as porcentagens de área dos picos correspondentes foram obtidos por um integrador Varian 4290. Os EMAGs foram tentativamente identificados pelo tempo de retenção de padrões de EMAGs (Sigma). Foi utilizado um cromatógrafo a gás acoplado a um detector de massas (CG-MS), Shimadzu QP 5000, com fragmentação por impacto de elétrons a $70 \mathrm{eV}$.

\section{Processo de metilação}

A transesterificação e esterificação foram realizadas utilizando-se o método de Joseph e Ackman. ${ }^{18}$

Em um tubo de ensaio com tampa rosqueável foi adicionado 1,0 mL de padrão interno (Pi) com concentração de $1 \mathrm{mg} / \mathrm{mL}$ de tricosanoato de metila (23:0Me) em iso-octano. Após a remoção completa do iso-octano com fluxo de $\mathrm{N}_{2}$ gasoso, aproximadamente $25,0 \mathrm{mg}$ de óleo foram adicionados ao tubo com o padrão interno e $1,5 \mathrm{~mL}$ de uma solução metanólica de $\mathrm{NaOH} 0,5 \mathrm{~mol} \mathrm{~L}^{-1}$, em seguida, a solução foi aquecida em banho-maria a $100{ }^{\circ} \mathrm{C}$ por $5 \mathrm{~min}^{-1} \mathrm{e}$ resfriada à temperatura ambiente. Dois $\mathrm{mL}$ de uma solução de $\mathrm{BF}_{3}$ (trifluoreto de boro) a $12 \%$ em metanol foram adicionados ao tubo, novamente colocado em banho-maria a $100{ }^{\circ} \mathrm{C}$ por $30 \mathrm{~min}^{-1} \mathrm{e}$ resfriado à temperatura ambiente. Após, adicionou-se 1,0 mL de iso-octano ao tubo, agitou-se vigorosamente por $30 \mathrm{~s} \mathrm{e}$, finalmente, adicionaramse 5,0 mL de solução aquosa saturada de $\mathrm{NaCl}$. A fase superior foi removida e transferida para um frasco âmbar de $5 \mathrm{~mL}$ e mais 1,0 $\mathrm{mL}$ de iso-octano foi adicionado. Após agitação, o sobrenadante foi retirado e adicionado à fração anterior que foi, então, concentrada para um volume final de, aproximadamente, $1,0 \mathrm{~mL}$ sob atmosfera de $\mathrm{N}_{2}$ gasoso.

\section{Fator de correção teórico $\left(\mathrm{F}_{\mathrm{CT}}\right)$}

A determinação do fator de correção teórico $\left(\mathrm{F}_{\mathrm{CT}}\right)$ é baseada no fato de que o (DIC) responde proporcionalmente à porcentagem em massa relativa à cadeia carbônica de um EMAG e que durante a combustão, o detector não responde "apreciavelmente" ao carbono do grupo carboxilato (COO). Desta forma, atribuindo-se, arbitrariamente, um fator teórico igual à unidade para um EMAG, por exemplo, para o estearato de metila (18:0Me), os fatores de correção para os demais ésteres metílicos podem ser encontrados, conforme expresso na Tabela 1. Desta forma, o $\mathrm{F}_{\mathrm{CT}}$ do DIC é uma constante para os diferentes EMAGs e não pode ser modificado devido a erros instrumentais ou de técnicas. ${ }^{12}$

\section{Determinação do fator de correção experimental $\left(\mathrm{F}_{\mathrm{CE}}\right)$}

Os fatores de correção experimental $\left(\mathrm{F}_{\mathrm{CE}}\right)$ do detector de ionização em chama (DIC) foram determinados em 6 replicatas, utilizando mistura de EMAGs (Sigma) incluindo os ésteres dos ácidos: láurico (12:0), miristico (14:0), palmítico (16:0), esteárico (18:0), oleico (18:1n-9), linoleico (18:2n-6), alfa-linolênico (18:3n-3), araquídico (20:0), araquidônico (20:4n-6), eicosapentaenoico (20:5n-3), behênico (22:0), docosahexaenoico (22:6n-3) e lignocérico (24:0) com quantidade conhecida de padrão interno $(\mathrm{Pi})$ tricosanoato de metila (23:0Me) e, a partir das áreas porcentuais e massas dos EMAGS e do $\mathrm{Pi}$, foi determinado o $\mathrm{F}_{\mathrm{CE}}$ para cada EMAG ${ }^{12,18}$ Os valores médios com os respectivos desvios padrão estão expressos na Tabela 2. A Equação 1 do $\mathrm{F}_{\mathrm{CE}}$ por definição é:

$$
F_{C E}=A_{p} / M_{p} \div A_{x} / M_{x}
$$


Tabela 1. Fator de correção teórico $\left(\mathrm{F}_{\mathrm{CT}}\right)$ para ésteres metílicos de ácidos graxos

\begin{tabular}{|c|c|c|c|c|c|c|c|}
\hline \multirow[b]{2}{*}{ *Éster metílico } & \multicolumn{7}{|c|}{ Ésteres metílicos de referência como padrão interno } \\
\hline & $\begin{array}{c}\text { 18:0Me Estearato } \\
\text { de metila }\end{array}$ & $\begin{array}{l}\text { 12:0Me Dode- } \\
\text { canoato de metila }\end{array}$ & $\begin{array}{l}\text { 17:0Me Heptade- } \\
\text { canoato de metila }\end{array}$ & $\begin{array}{l}\text { 18:1Me Oleato } \\
\text { de metila }\end{array}$ & $\begin{array}{l}\text { 19:0Me Nonade- } \\
\text { canoato de metila }\end{array}$ & $\begin{array}{l}\text { 21:0Me Heneico- } \\
\text { sanoato de metila }\end{array}$ & $\begin{array}{r}\text { 23:0Me Tricosa- } \\
\text { noato de metila }\end{array}$ \\
\hline 4:0 & 1,5396 & 1,4294 & 1,5257 & 1,5501 & 1,5522 & 1,5742 & 1,5930 \\
\hline $5: 0$ & 1,4009 & 1,3006 & 1,3883 & 1,4105 & 1,4123 & 1,4324 & 1,4495 \\
\hline $6: 0$ & 1,3084 & 1,2147 & 1,2966 & 1,3174 & 1,3191 & 1,3378 & 1,3538 \\
\hline 7:0 & 1,2423 & 1,1534 & 1,2311 & 1,2508 & 1,2524 & 1,2702 & 1,2854 \\
\hline $8: 0$ & 1,1927 & 1,1073 & 1,1820 & 1,2009 & 1,2024 & 1,2195 & 1,2340 \\
\hline 9:0 & 1,1542 & 1,0716 & 1,1438 & 1,1621 & 1,1636 & 1,1802 & 1,1942 \\
\hline 10:0 & 1,1233 & 1,0429 & 1,1132 & 1,1310 & 1,1325 & 1,1486 & 1,1622 \\
\hline 11:0 & 1,0981 & 1,0195 & 1,0882 & 1,1056 & 1,1071 & 1,1228 & 1,1361 \\
\hline $12: 0$ & 1,0771 & 1,0000 & 1,0674 & 1,0845 & 1,0859 & 1,1013 & 1,1144 \\
\hline $12: 1$ & 1,0670 & 0,9906 & 1,0574 & 1,0743 & 1,0757 & 1,0910 & 1,1040 \\
\hline $13: 0$ & 1,0593 & 0,9835 & 1,0497 & 1,0666 & 1,0680 & 1,0831 & 1,0960 \\
\hline $14: 0$ & 1,0441 & 0,9694 & 1,0347 & 1,0512 & 1,0526 & 1,0676 & 1,0803 \\
\hline $14: 1$ & 1,0354 & 0,9613 & 1,0261 & 1,0425 & 1,0439 & 1,0587 & 1,0713 \\
\hline $15: 0$ & 1,0308 & 0,9570 & 1,0215 & 1,0379 & 1,0392 & 1,0540 & 1,0665 \\
\hline $15: 1$ & 1,0227 & 0,9495 & 1,0135 & 1,0297 & 1,0311 & 1,0457 & 1,0581 \\
\hline $16: 0$ & 1,0193 & 0,9463 & 1,0101 & 1,0263 & 1,0276 & 1,0422 & 1,0546 \\
\hline $16: 1$ & 1,0117 & 0,9393 & 1,0026 & 1,0186 & 1,0200 & 1,0345 & 1,0468 \\
\hline $16: 2$ & 1,0041 & 0,9322 & 0,9951 & 1,0110 & 1,0123 & 1,0267 & 1,0389 \\
\hline $16: 3$ & 0,9965 & 0,9252 & 0,9875 & 1,0033 & 1,0046 & 1,0189 & 1,0310 \\
\hline $16: 4$ & 0,9889 & 0,9181 & 0,9799 & 0,9957 & 0,9970 & 1,0111 & 1,0232 \\
\hline $17: 0$ & 1,0091 & 0,9369 & 1,0000 & 1,0160 & 1,0173 & 1,0318 & 1,0440 \\
\hline $17: 1$ & 1,0019 & 0,9302 & 0,9929 & 1,0088 & 1,0101 & 1,0244 & 1,0366 \\
\hline 18:0 & 1,0000 & 0,9284 & 0,9910 & 1,0068 & 1,0082 & 1,0225 & 1,0347 \\
\hline $18: 1$ & 0,9932 & 0,9221 & 0,9842 & 1,0000 & 1,0013 & 1,0155 & 1,0276 \\
\hline $18: 2$ & 0,9865 & 0,9159 & 0,9776 & 0,9933 & 0,9946 & 1,0087 & 1,0207 \\
\hline $18: 3$ & 0,9797 & 0,9096 & 0,9709 & 0,9864 & 0,9877 & 1,0017 & 1,0137 \\
\hline $18: 4$ & 0,9730 & 0,9034 & 0,9642 & 0,9797 & 0,9809 & 0,9949 & 1,0067 \\
\hline 19:0 & 0,9919 & 0,9209 & 0,9830 & 0,9987 & 1,0000 & 1,0142 & 1,0263 \\
\hline 20:0 & 0,9846 & 0,9141 & 0,9757 & 0,9913 & 0,9926 & 1,0067 & 1,0187 \\
\hline 20:1 & 0,9785 & 0,9085 & 0,9697 & 0,9852 & 0,9865 & 1,0005 & 1,0124 \\
\hline $20: 2$ & 0,9724 & 0,9028 & 0,9636 & 0,9791 & 0,9803 & 0,9943 & 1,0061 \\
\hline $20: 3$ & 0,9663 & 0,8971 & 0,9576 & 0,9729 & 0,9742 & 0,9880 & 0,9998 \\
\hline $20: 4$ & 0,9603 & 0,8916 & 0,9516 & 0,9669 & 0,9681 & 0,9819 & 0,9936 \\
\hline $20: 5$ & 0,9542 & 0,8859 & 0,9456 & 0,9607 & 0,9620 & 0,9757 & 0,9878 \\
\hline 21:0 & 0,9780 & 0,9080 & 0,9692 & 0,9847 & 0,9860 & 1,0000 & 1,0119 \\
\hline $22: 0$ & 0,9720 & 0,9024 & 0,9632 & 0,9787 & 0,9799 & 0,9939 & 1,0057 \\
\hline $22: 1$ & 0,9664 & 0,8972 & 0,9577 & 0,9730 & 0,9743 & 0,9881 & 0,9999 \\
\hline $22: 2$ & 0,9609 & 0,8921 & 0,9522 & 0,9675 & 0,9687 & 0,9825 & 0,9942 \\
\hline $22: 3$ & 0,9554 & 0,8870 & 0,9468 & 0,9619 & 0,9632 & 0,9769 & 0,9885 \\
\hline $22: 4$ & 0,9499 & 0,8819 & 0,9413 & 0,9564 & 0,9577 & 0,9713 & 0,9828 \\
\hline $22: 5$ & 0,9443 & 0,8767 & 0,9358 & 0,9508 & 0,9520 & 0,9655 & 0,9770 \\
\hline $22: 6$ & 0,9388 & 0,8716 & 0,9303 & 0,9452 & 0,9465 & 0,9599 & 0,9713 \\
\hline $23: 0$ & 0,9665 & 0,8973 & 0,9578 & 0,9731 & 0,9744 & 0,9882 & 1,0000 \\
\hline $24: 0$ & 0,9615 & 0,8927 & 0,9528 & 0,9681 & 0,9694 & 0,9831 & 0,9948 \\
\hline $24: 1$ & 0,9564 & 0,8879 & 0,9478 & 0,9629 & 0,9642 & 0,9779 & 0,9896 \\
\hline
\end{tabular}

*A simbologia representa a cadeia principal do EMAG. Foram consideradas as seguintes massas atômicas: $\mathrm{C}=12,0110 ; \mathrm{H}=1,0079 ; \mathrm{O}=15,9994$. Os isômeros dos EMAGs posicionais e geométricos e os ésteres metílicos ramificados apresentam o mesmo $\mathrm{F}_{\mathrm{CT}}$ desde que apresentem o mesmo número de $\mathrm{C}^{*}$. 
Fazendo o rearranjo temos:

$$
F_{C E}=A_{p} \times M_{x} \div M_{p} \times A_{x}
$$

onde: $\mathrm{A}_{\mathrm{P}}=$ área do padrão; $\mathrm{M}_{\mathrm{P}}=$ massa do padrão; $\mathrm{A}_{\mathrm{X}}=$ área do EMAG X e $\mathrm{M}_{\mathrm{X}}=$ massa do EMAG X.

Tabela 2. Fator de resposta experimental e teórico em relação ao padrão interno $(\mathrm{Pi})$ metil tricosanoato $(23: 0 \mathrm{Me})$

\begin{tabular}{lccc}
\hline \multirow{2}{*}{ EMAGs } & \multicolumn{2}{c}{ Fator de resposta } & Coeficiente $(\mathrm{C})$ \\
& Experimental $\left(\mathrm{F}_{\mathrm{CE}}\right)^{\mathrm{a}}$ & Theoretical $\left(\mathrm{F}_{\mathrm{CT}}\right)^{\mathrm{b}}$ & $\mathrm{C}=\mathrm{F}_{\mathrm{CE}} / \mathrm{F}_{\mathrm{CT}}$ \\
\hline $12: 0 \mathrm{Me}$ & $1,0535 \pm 0,0095$ & 1,1144 & 0,9454 \\
$14: 0 \mathrm{Me}$ & $1,0653 \pm 0,0093$ & 1,0803 & 0,9861 \\
$16: 0 \mathrm{Me}$ & $1,0491 \pm 0,0092$ & 1,0546 & 0,9948 \\
$18: 0 \mathrm{Me}$ & $1,0282 \pm 0,0094$ & 1,0347 & 0,9937 \\
$18: 1 \mathrm{n}-9 \mathrm{Me}$ & $1,0329 \pm 0,0098$ & 1,0276 & 1,0052 \\
$18: 2 \mathrm{n}-6 \mathrm{Me}$ & $1,0524 \pm 0,0189$ & 1,0207 & 1,0311 \\
$18: 3 \mathrm{n}-3 \mathrm{Me}$ & $1,0505 \pm 0,0168$ & 1,0137 & 1,0363 \\
$20: 0 \mathrm{Me}$ & $1,0274 \pm 0,0083$ & 1,0187 & 1,0085 \\
$20: 4 \mathrm{n}-6 \mathrm{Me}$ & $1,0484 \pm 0,0198$ & 0,9936 & 1,0552 \\
$20: 5 \mathrm{n}-3 \mathrm{Me}$ & $1,0443 \pm 0,0239$ & 0,9878 & 1,0572 \\
$22: 0 \mathrm{Me}$ & $0,9905 \pm 0,0092$ & 1,0057 & 0,9849 \\
$22: 6 \mathrm{n}-3 \mathrm{Me}$ & $1,0442 \pm 0,0278$ & 0,9713 & 1,0751 \\
$23: 0 \mathrm{Me}$ & $1,0000 \pm 0,0000$ & 1,0000 & 1,0000 \\
$24: 0 \mathrm{Me}$ & $0,9874 \pm 0,0104$ & 0,9948 & 0,9926 \\
\hline
\end{tabular}

avalores médios \pm estimativas dos desvios padrão $(\mathrm{n}=6)$, ${ }^{\mathrm{b}}$ ref.19, EMAGs Ésteres metílicos de ácidos graxos.

\section{RESULTADOS E DISCUSSÃO}

Determinação do fator de correção teórico $\left(F_{\mathrm{CT}}\right)$ do detector de ionização em chama

\section{Cálculo da porcentagem de carbono ativo ( $\left.C^{*}\right)$}

No intuito de ilustrar a aplicação do método de determinação da porcentagem de carbono ativo $\left(\mathrm{C}^{*}\right)$ e o fator de correção teórico $\left(\mathrm{F}_{\mathrm{CT}}\right)$ do detector de ionização em chama, foi utilizado como padrão interno (Pi) o estearato de metila $\left(\mathrm{C}_{19} \mathrm{H}_{38} \mathrm{O}_{2}\right.$, massa molar de 298,5080) no cálculo do fator do decanoato de metila $\left(\mathrm{C}_{11} \mathrm{H}_{22} \mathrm{O}_{2}\right.$, massa molar de 186,2936). É importante lembrar que o grupo COO (massa molar de 44,0098) do EMAG apresenta resposta desprezível no DIC e, considerando somente os carbonos ativos $\left(\mathrm{C}^{*}\right)$ nas moléculas de EMAGs, tem-se:

Estearato de metila $=18 C^{*} \times 12,0110=216,1980$,

Decanoato de metila $=10 C^{*} \times 12,0110=120,1100$.

Portanto, a porcentagem relativa para cada EMAG será:

Estearato de metila $298,5080(100 \%)$ e $216,1980\left(\%\right.$ de $\left.C^{*}\right)=$ $72,4262 \%$ de $C^{*}$

Decanoato de metila $186,2936(100 \%)$ e $120,1100\left(\%\right.$ de $\left.C^{*}\right)=$ $64,4735 \%$ de C*

Dividindo-se a $\%$ de $C^{*}$ do estearato de metila pela $\%$ de $C^{*}$ do decanoato de metila:
$F_{C T}$ do decanoato de metila $+72,4262 \% \div 64,4735 \%=1,1233$

Desta forma, o valor de 1,1233 deverá ser utilizado como $\mathrm{F}_{\mathrm{CT}}$ na determinação quantitativa do decanoato de metila (10:0Me) para corrigir a resposta diferencial do DIC e com o estearato de metila como Pi. Valores para outros EMAGs estão expressos na Tabela 1.

É possível de maneira simplificada obter novos valores do $\mathrm{F}_{\mathrm{CT}}$ dos EMAGs utilizando outro padrão interno (Pi), basta utilizar os valores obtidos para o metil estearato como Pi (primeira coluna - Tabela 1) e os valores do novo padrão interno pretendido. Por exemplo, para encontrar o novo valor de $\mathrm{F}_{\mathrm{CT}}$ metil éster do ácido alfa-linolênico (18:3Me) utilizando agora como Pi o tricosanoato de metila (23:0Me), basta simplesmente dividir os valores de $\mathrm{F}_{\mathrm{CT}}$ do $18: 3 \mathrm{Me}$ pelo $\mathrm{F}_{\mathrm{CT}}$ do Pi (23:0Me), conforme $\mathrm{F}_{\mathrm{CT}}$ da Tabela 1, o cálculo é mostrado na Equação 2.

$$
F_{C T} \text { do } 18: 3=\frac{F_{C T} 18: 3}{F_{C T} 23: 0}=\frac{0,9797}{0,9665}=1,0137
$$

A Tabela 1 apresenta o $\mathrm{F}_{\mathrm{CT}}$ para vários EMAGs com diferentes padrões internos, $\mathrm{Pi}$, estearato de metila (18:0Me), dodecanoato de metila $(12: 0 \mathrm{Me})$, heptadecanoato de metila $(17: 0 \mathrm{Me})$, oleato de metila (18:1Me), nonadecanoato de metila (19:0Me), heneicosanoato de metila (21:0Me) e tricosanoato de metila (23:0Me). Todos os resultados apresentados na Tabela 1 estão de acordo com os valores publicados na literatura. $^{25}$

\section{Determinação do fator de correção experimental $\left(\mathrm{F}_{\mathrm{CE}}\right)$ do detector de ionização em chama}

Os resultados das determinações dos fatores experimentais $\left(\mathrm{F}_{\mathrm{CE}}\right)$ foram determinados utilizando a Equação1 e os resultados estão expressos na Tabela 2.

Neste estudo, foram utilizados os dados da Tabela 1, empregandose o metil tricosanoato (23:0Me) como padrão interno.

De maneira geral, os coeficientes $(\mathrm{C})$ entre os fatores de resposta experimental, $\mathrm{F}_{\mathrm{CE}}$, e teórica, $\mathrm{F}_{\mathrm{CT}}$, dos EMAGs saturados (Tabela 2) são mais próximos da unidade em relação aos poli-insaturados. Desta forma, recomenda-se o uso de um EMAG saturado como padrão interno e os resultados estão em concordância com os expressos na literatura. ${ }^{12} \mathrm{O}$ ideal é obter coeficiente igual à unidade, assim, pode-se afirmar que o equipamento está operando em condições otimizadas. Por outro lado, um coeficiente muito elevado para um ácido graxo saturado dá uma indicação de que o equipamento não está otimizado e/ou existem falhas na técnica. Apesar dos ácidos graxos saturados serem mais estáveis do que os insaturados, recomenda-se nos métodos de quantificação o uso de padrões novos, preparados e armazenados adequadamente. $^{12}$

\section{Determinação da concentração de ácidos graxos em uma amostra de óleo de peixe}

A massa expressa em mg de EMAG foi determinada em função da massa do EMAG $\left(\mathbf{M}_{\mathrm{x}}\right.$, incógnita desejada), conforme a Equação 1. No entanto, para aumentar o grau de confiança na quantificação de EMAGs ou ácidos graxos, alguns autores recomendam o uso do fator de correção teórico $\left(\mathrm{F}_{\mathrm{CT}}\right)$, desde que os parâmetros químicos e instrumentais também estejam otimizados, para que os erros oriundos dessa fonte sejam eliminados. Alguns autores afirmaram que, devido à instabilidade oxidativa dos ácidos graxos poli-insaturados, é virtualmente impossível obter e manter padrões deste tipo, com alto grau de pureza e recomendaram como abordagem apropriada para análises de poli-insaturados o uso de fator de correção teórico $\left(\mathrm{F}_{\mathrm{CT}}\right)$. 
Colocando a Equação 1 em função da massa de um ácido graxo $\mathrm{X}$ tem-se a Equação 3.

$$
M_{x}=M_{p} \times A_{x} \times F_{C T} / A_{p}
$$

\section{Equação final e fator de conversão de EMAG (éster metílico)} para ácido graxo

Nas análises de alimentos, os resultados devem ser expressos em ácidos graxos, diferentemente do biodiesel em que os resultados devem ser expressos em EMAG. Portanto, ainda se tem que adicionar à Equação 3 a quantidade de amostra $\left(\mathrm{M}_{\mathrm{A}}\right)$ e o fator de conversão de éster metílico para ácido graxo $\left(\mathrm{FC}_{\mathrm{EA}}\right)$, agora denominada de equação final para determinação de ácidos graxos em $\mathrm{mg} / \mathrm{g}$ de óleo ou gordura. O FCEA é determinado dividindo-se a massa molecular do EMAG pela massa molecular do ácido graxo correspondente, conforme mostra a Equação 4:

$$
F_{C E A}=\frac{\text { Massa molecular do EMAG }}{\text { Massa molecular do ácido graxo }}
$$

O EMAG de um determinado ácido graxo apresenta o grupo metil $\left(\mathrm{CH}_{3}\right)$ em substituição ao átomo de $\mathrm{H}$.

$$
\mathrm{R}-\mathrm{COOCH3} \rightarrow \mathrm{R}-\mathrm{COOH}
$$

Assim, o éster metílico apresentará maior resposta no DIC em relação ao ácido graxo correspondente, pois o $\left(\mathrm{CH}_{3}\right)$ do EMAG contribui para aumentar o número de $\mathrm{C}^{*}$ em relação ao ácido graxo.

A Tabela 3 mostra a massa molecular (MM) e o valor do $F_{\text {CEA }}$ para alguns ésteres metílicos e seus respectivos ácidos graxos.

No caso da determinação de biodiesel (EMAGs), o $\mathrm{F}_{\mathrm{CEA}}$ deverá ser igual a 1, pois no biodiesel o resultado é expresso em EMAG e não em ácidos graxos. No entanto, na expressão em ácidos graxos deveremos utilizar o $\mathrm{F}_{\mathrm{CEA}}$, conforme a equação final.

$$
M_{x}=M_{p} \times A_{x} \times F_{C T} / A_{p} \times M_{A} \times F_{C E A}
$$

onde: $\mathrm{M}_{\mathrm{P}}=$ massa do padrão interno (Pi) em mg; $\mathrm{A}_{\mathrm{X}}=$ área do EMAG $\mathrm{X} ; \mathrm{F}_{\mathrm{CT}}=$ fator de correção teórico; $\mathrm{A}_{\mathrm{P}}=$ área do padrão interno; $\mathrm{M}_{\mathrm{A}}$ = massa da amostra (óleo ou gordura) em $\mathrm{g} ; \mathrm{M}_{\mathrm{X}}=$ massa do ácido graxo $\mathrm{X}$ em mg/g de óleo ou gordura; $\mathrm{F}_{\mathrm{CEA}}=$ fator de conversão éster metílico para ácido graxo.
Tabela 3. Fator de conversão éster metílico para ácido graxo $F_{\text {CEA }}$

\begin{tabular}{lccc}
\hline Ácidos graxos & MM do AG MM do EMAG & $\mathrm{F}_{\text {CEA }}$ \\
\hline Tetradecanoico-14:0 $\left(\mathrm{C}_{14} \mathrm{H}_{28} \mathrm{O}_{2}\right)$ & 228 & 242 & 1,061 \\
Hexadecanoico-16:0 $\left(\mathrm{C}_{16} \mathrm{H}_{32} \mathrm{O}_{2}\right)$ & 256 & 270 & 1,055 \\
Octadecanoico- 18:0 $\left(\mathrm{C}_{18} \mathrm{H}_{36} \mathrm{O}_{2}\right)$ & 284 & 298 & 1,049 \\
9- Octadecenoico-18:1n-9 $\left(\mathrm{C}_{18} \mathrm{H}_{34} \mathrm{O}_{2}\right)$ & 282 & 296 & 1,050 \\
9,12-Octadienoico-18:2n-6 $\left(\mathrm{C}_{18} \mathrm{H}_{32} \mathrm{O}_{2}\right)$ & 280 & 296 & 1,057 \\
9,12,15-Octadecatrienoico- & 278 & 292 & 1,050 \\
18:3n-3 $\left(\mathrm{C}_{18} \mathrm{H}_{30} \mathrm{O}_{2}\right)$ & & & \\
Eicosanoico-20:0 $\left(\mathrm{C}_{20} \mathrm{H}_{40} \mathrm{O}_{2}\right)$ & 312 & 326 & 1,045 \\
5,8,11,14-Eicosatetraenoico- & 304 & 318 & 1,046 \\
20:4n-6 $\left(\mathrm{C}_{18} \mathrm{H}_{32} \mathrm{O}_{2}\right)$ & & & \\
5,8,11,14,17-Eicosapentaenoico- & 302 & 316 & 1,044 \\
20:5n-3 $\left(\mathrm{C}_{20} \mathrm{H}_{30} \mathrm{O}_{2}\right)$ & & & \\
Docosanoico-22:0 $\left(\mathrm{C}_{22} \mathrm{H}_{44} \mathrm{O}_{2}\right)$ & 340 & 354 & 1,041 \\
4,7,10,13,16,19 - Docosahexaenoico- & 328 & 342 & 1,042 \\
22:6n-3 $\left(\mathrm{C}_{22} \mathrm{H}_{32} \mathrm{O}_{2}\right)$ & & & \\
\hline $\begin{array}{l}\text { Foram consideradas as seguintes massas atômicas: } \mathrm{H}=1 ; \mathrm{C}=12 \mathrm{e} \mathrm{O}=16 . \mathrm{MM} \\
\text { - massa molecular, AG - ácido graxo, EMAG - éster metílico de ácido graxo. }\end{array}$
\end{tabular}

Concentração de ácidos graxos expressos em quantidade de ácidos graxos em uma amostra de peixe

Nas amostras do óleo de tilápia, foi estudado um total de 11 ácidos graxos, conforme a Tabela 4. Estes ácidos são os mais importantes sob o ponto de vista tecnológico e como bioativos funcionais para a saúde humana. ${ }^{26} \mathrm{Na}$ obtenção da concentração em miligramas de ácido graxo por grama de óleo (Tabela 4) foi utilizada a equação final:

$$
M_{x}=M_{p} \times A_{x} \times F_{C T} / A_{p} \times M_{A} \times F_{C E A}
$$

O teor médio de óleo (lipídios totais) encontrado nos filés foi de $1,1 \%$ e o teor de umidade de $76,9 \%$. Estes valores são similares àqueles encontrados por outros pesquisadores para tilápias. ${ }^{5}$

Na Tabela 4, observam-se valores expressos pelo método da normalização (porcentagem de área relativa). Quando os resultados são utilizados na área de alimentos, os resultados são expressos de uma

\begin{tabular}{|c|c|c|c|c|}
\hline \multirow[t]{2}{*}{ Ácidos graxos } & $\begin{array}{l}\text { Porcentagem de área relativa } \\
\text { (área do ácido graxo/ área total) }\end{array}$ & $\begin{array}{l}\text { Fator de correção } \\
\text { teórico }\left(\mathrm{F}_{\mathrm{CT}}\right)\end{array}$ & $\begin{array}{c}\text { Fator de conversão } \\
\text { EMAG para Ácido }\left(\mathrm{F}_{\mathrm{CEA}}\right)\end{array}$ & $\begin{array}{c}\text { Concentração (mg de ácido } \\
\text { graxo/g de óleo) }\end{array}$ \\
\hline & I & II & III & IV \\
\hline $14: 0$ & $0,7 \pm 0,1$ & 1,0803 & 1,055 & $4,1 \pm 0,4$ \\
\hline $16: 0$ & $14,3 \pm 0,4$ & 1,0546 & 1,061 & $87,2 \pm 5,2$ \\
\hline 18:0 & $6,1 \pm 0,7$ & 1,0347 & 1,049 & $16,5 \pm 1,4$ \\
\hline $18: 1 \mathrm{n}-9$ & $20,0 \pm 1,1$ & 1,0276 & 1,050 & $128,0 \pm 9,8$ \\
\hline $18: 2 n-6$ & $20,7 \pm 1,1$ & 1,0207 & 1,057 & $138,7 \pm 10,1$ \\
\hline $18: 3 n-3$ & $10,6 \pm 1,4$ & 1,0137 & 1,050 & $59,3 \pm 7,5$ \\
\hline $20: 0$ & $0,2 \pm 0,1$ & 1,0187 & 1,045 & $1,4 \pm 0,2$ \\
\hline $20: 4 n-6$ & $2,6 \pm 0,3$ & 0,9936 & 1,046 & $13,0 \pm 1,3$ \\
\hline $20: 5 n-3$ & $0,5 \pm 0,1$ & 0,9878 & 1,044 & $2,5 \pm 0,4$ \\
\hline $22: 0$ & $0,1 \pm 0,1$ & 1,0057 & 1,042 & $6,5 \pm 0,5$ \\
\hline $22: 6 n-3$ & $5,1 \pm 0,2$ & 0,9713 & 1,042 & $26,1 \pm 2,0$ \\
\hline
\end{tabular}
forma bem simplificada, em porcentagem de área relativa ou massa porcentual de cada ácido graxo pela massa total de ácidos graxos, conforme mostra a coluna I da Tabela 4. Na verdade, o DIC responde

Tabela 4. Concentração de ácidos graxos em mg/g de óleo de tilápia 
em massa e o correto é expressar a massa porcentual de cada éster metílico/massa total de ésteres metílicos presente no cromatograma. No entanto, alguns autores expressam erroneamente as concentrações em mg ou g de ácidos graxos/100 g de óleo ou gordura e esses resultados geram muita confusão nas interpretações.

Deve-se lembrar que em $100 \mathrm{~g}$ de óleo e na dependência da natureza têm-se vários constituintes, como fosfolipídios, vitaminas lipossolúveis, esteróis, pigmentos, glicerol resultante da reação de transesterificação etc., portanto, $100 \mathrm{~g}$ de óleo ou gordura não apresentam $100 \%$ de ácidos graxos.

$\mathrm{Na}$ Tabela 4, o $\mathrm{F}_{\mathrm{CT}}$ (coluna II) foi utilizado na obtenção da concentração de ácidos graxos. Este fator tem sido recomendado ${ }^{18,25}$ pela sua praticidade e sempre que as condições metodológicas estiverem otimizadas. $\mathrm{O}$ fator de conversão EMAG para ácido graxo $\mathrm{F}_{\mathrm{CEA}}$ (coluna III) deve ser utilizado, considerando que em alimentos os resultados são expressos em ácidos graxos e não em EMAGs. ${ }^{12}$

A influência no resultado da concentração de ácidos graxos em mg de ácido graxo/g de óleo foi avaliada removendo-se da equação final os valores do fator de correção teórico $\left(\mathrm{F}_{\mathrm{CT}}\right)$, ou seja, realizando os cálculos da concentração de ácidos graxos sem o $\mathrm{F}_{\mathrm{CT}}$. As diferenças entre os resultados foram superiores para os ácidos graxos de cadeia curta 14:0 (7,3\%), 16:0 (5,3\%) e 18:0 (3,65\%), baixos para os ácidos $20: 4 n-6(0,8 \%)$ e $18: 3 n-3(1,3 \%)$ e praticamente inexistentes para os ácidos com baixa concentração em ácidos graxos 20:0, 22:0 e 20:5n-3.

As concentrações dos ácidos graxos em $\mathrm{mg} / \mathrm{g}$ de óleo estão dispostas na Tabela 4. Esses resultados são facilmente interpretados e transformados em diferentes expressões, por exemplo, como os filés de tilápias apresentam $1,1 \%$ de óleo e os resultados da Tabela 4 são expressos por g de óleo (coluna IV), ao multiplicar o valor da concentração por 1,1 (porcentagem de óleo no filé) obtem-se o a concentração em mg de ácidos graxos/100 g de filé. Desta forma, os resultados obtidos são facilmente utilizados para a elaboração de tabelas alimentares, rótulos e nas interpretações e discussões quantitativas nos artigos científicos entre matérias-primas.

\section{CONCLUSÕES}

As ilustrações, instruções e resultados apresentados com o uso destas técnicas analíticas mostraram que é possível aumentar a exatidão na expressão da concentração de ácidos graxos (em alimentos) ou ésteres de ácidos graxos (em biodiesel), principalmente, quando comparados com o método da normalização de área, um método limitado e que muitas vezes é utilizado erroneamente. Além disso, os resultados das análises utilizando padronização interna e fatores de correção, em uma amostra de óleo de peixe, mostraram que a expressão dos resultados da concentração, em massa de ácido graxo por massa de óleo ou de matéria-prima, deve ser sempre utilizada. Assim, os resultados obtidos são confiáveis e facilmente interpretados, permitindo comparações quantitativas da composição de ésteres de ácidos graxos nos alimentos e em biodiesel.

\section{REFERÊNCIAS}

1. Suarez, P. A. Z.; Meneghetti, S. M. P.; Wolf, C. R.; Quim. Nova 2007, 30,667 .

2. Suarez, P. A. Z.; Meneghetti, S. M. P.; Quim. Nova 2007, 30, 2068.

3. Prado, I. N.; Prado, R. M.; Rotta, P. P.; Visantainer, J. V.; Moletta, J. L.; Perotto, D.; J. Anim. Feed Sci. 2008, 17, 295.

4. Prado, I. N.; Ito, R. H.; Prado, J. M.; Prado, I. M.; Rotta, P. P.; Matsushita, M.; Visentainer, J. V.; Silva, R. R.; J. Anim. Feed Sci. 2008, 17, 307.

5. Tonial, I. B.; Stevanato, F. B.; Matsushita, M.; De Souza, N. E.; Furuya, W. M.; Visentainer, J. V.; Aquacult. Nutr. 2009, 15, 564.

6. Ruiz, M. R.; Matsushita, M.; Visentainer, J. V.; Hernandez, J. A.; Ribeiro, E. L. D.; Shikomaki, M.; Reeves, J. J.; de Souza, N. E.; S. Afr. J. Anim. Sci. 2005, 35, 13 .

7. Tanamati, A.; Stevanato, F. B.; Visentainer, J. E. L.; Matsushita, M.; de Souza, N. E.; Visentainer, J. V.; Eur. J. Lipid Sci. Technol. 2009, 111, 183.

8. Martin, C. A.; Milinsk, M. C.; Visentainer, J. V.; Matsushita, M.; DeSouza, N. E.; An. Acad. Bras. Ciênc. 2007, 79, 343.

9. Martin, C. A.; Carapelli, R.; Visantainer, J. V.; Matsushita, M.; de Souza, N. E.; Food Chem. 2005, 93, 445

10. Oliveira, F. C. C.; Suarez, P. A. Z.; Santos, W. L. P.; Química Nova na Escola 2008, n. 28, 3.

11. Marques, M. V.; Naciuk, F. F.; Mello, A. M. S.; Seibel, N. M.; Fontoura, L. A. M.; Quim. Nova 2010, 33, 978.

12. Visentainer, J. V.; Franco, M. R. B.; Ácidos Graxos em Óleos e Gorduras: Identificação e Quantificação, $1^{\mathrm{a}}$ ed, Varela: São Paulo, 2006.

13. Collins, C. H.; Braga, G. L.; Bonato, P. S.; Fundamentos de Cromatografia, Ed. da Unicamp: Campinas, 2006.

14. Holm, T.; J. Chromatogr., A 1999, 842, 221.

15. Christie, W. W.; Gas Chromatography and lipids - practical guide, $1^{\mathrm{st}}$ ed., The Oil Press LTd: Dundee, 1994.

16. Ackman, R. G.; Prog. Chem. Fats Lip. 1972, 12, 165.

17. Ackman, R.; Sipos, J. C.; JAOCS 1964, 41, 377.

18. Joseph, J. D.; Ackman, R. G.; J. AOAC Int. 1992, 75, 488.

19. Visentainer, J. V.; Noffs, M. D.; Carvalho, O. D. O.; de Almeida, V. V.; de Oliveira, C. C.; de Souza, N. E.; JAOCS 2007, 84, 543.

20. Tarley, C. R. T.; Visentainer, J. V.; Matsushita, M.; de Souza, N. E.; Food Chem. 2004, 88, 1.

21. Aguiar, A. C.; Cottica, S. M.; Boroski M.; Oliveira, C. C.; Bonafe, E. G.; França, P. B.; Souza, N. E.; Visentainer, J. V.; J. Braz. Chem. Soc. 2011, 22,643 .

22. Bligh, E. G.; Dyer, W.; Can. J. Biochem. Physiol. 1959, 37, 911.

23. AOAC; Official Methods of Analysis, $15^{\text {th }}$ ed., Association of Official Analytical Chemistis: Arlington, 1990.

24. Martin, C. A.; de Oliveira, C. C.; Visentainer, J. V.; Matsushita, M.; de Souza, N. E.; J. Chromatogr., A 2008, 1194, 111.

25. Craske, J. D.; Bannon, C. D.; JAOCS 1988, 65, 1190; Visentainer, J. V.; de Souza, N. E.; Makoto, M.; Hayashi, C.; Franco, M. R. B.; Food Chem. 2005, 90, 557.

26. Aguiar, A. C.; Morais, D. R.; Santos, L. P.; Stevanato, F. B.; Visentainer, J. E. L.; de Souza, N. E.; Visentainer, J. V.; Arch. Latinoam. Nutr. 2007, $57,273$. 